\title{
Acknowledgment to Referees
}

(September 1,1994 -November 8,1995)

The editors wish to express their sincere appreciation to the numerous colleagues who havecritically assessed submitted papers thereby providing the information indispensable for maintaining the scientific standing of Caries Research. J. Tenovuo

A. S. AaltonenS. AlaluusuaP. Alanen

P. Anderson

B. Angmar-MånssonJ. Arenas

T. Arnebrant

F. P. Ashley

V Baelum

J. Bánóczy

J.W. Bawden

J.D. Beck

D. Beighton

D.K. Benn

R. Billings

J.M. Birkeland

D. Birkhed

J. M. P. M. Borggreven

J. J. ten Bosch

P. Bottenberg

G. BowdenW.H. BowenD. BratthallH. J. BusscherW.T.Butler

J. Carlsson J.M. tenCate P. Caufield B.H. Clarkson A.L. Coykendall R. E. Corpron S.L. Creanor

M. Curzon T.W. Cutress

G. Dahlén

C. DawesT.G. DeatonG.H.DibdinJ. van DijkenA.G. DijkmanJ.A. DisneyH.

DonoghueC.W.I. DouglasM.C. Downer

R. M. Duckworth M.S. Duggal H. Duschner

WM. Edgar S. Edwardsson A.D. Eisenberg J. Ekstrand K. Ekstrand R.J. Elderton J.C.Elliott

C.G. Emilson G. Embery H.M. Eriksen I. Espelid

J.D.B. Featherstone F. von der Fehr

A. R. FirestoneH. Forss

L. Forsten R.M. Frank S. Fure

D.A.M.Geddes E. Giertsen A.S.M. Gilmour P. Gjermo P.O. Glantz T.H. Grenby S.R.Grobler H.

Gröndahl

B. Guggenheim

I.R.Hamilton

CM. Hanes

M. Hannig 

J.M. Hardie
D.S. Harper
J.C. Hase
H. Hausen
D. Hay
T.J. Heaven
A. F. Hefti
H. Hintze
J. S. vanderHoeven
W.P.Holbrook

A. K. Holm

D. Hoist

E. HonkalaJ. van HouteP. Hyvönen

T. Imfeld G. S. Ingram

I. Johansson D.C. Johnsen W. J. Jongebloed E. de Josselin de Jong

S. Kalfas S. Karjalainen S. Kashket E. Kerosuo E.A.M.Kidd M. Kilian

E. KirkegaardI. KleinbergB. Klock

M. Knuuttila G. Koch B. Krasse

B. KöhlerK.G.König

F. LagerlöfM. LarmasM. J. LarsenR.Z. LeGerosM. J. LevineS.M. Levy

P. Lingström W.J. Loesche

C. LongbottomH. Luoma
A. Lussi
C. van Loveren
I.D. Mandel

F. Manji R. H. Manning H.C. Margolis P. D. Marsh T. Marthaler J.R. Mellberg J.H. Meurman S.M. Mickhalek LA. Mjör E.C. Moreno T. Modeer

C. Mouton

B. Mânsson-Rahemtualla S. A. Mundorff-Shrestha H. Murtomaa K.K. Mäkinen J.M. Navia T Nederfors D.G. A. Nelson E. Newbrun
A. V Nieuw AmerongenP. V Nummikoski
B. Nyvad
B. ØgaardT OkanoA. OlivebyJ. Olsson
D. O’MullaneT. Ooshíma
D. Ørstavik
W van Palenstein-Helderman I. Paunio
E. Pearce

L. G. Peters son K. Pienihäkkinen N. Pitts K.M. Pruitt

M. Raadal H.R. Rawls E. C. Reynolds P.J. Riordan L.W Ripa

C. RobinsonJ. Rudney

A.J. Rugg-Gunn J. Rundegren A.D.Russell M. Russell R. R. Russell I. Rytömaa G. Rølla H.J. Sandham C.F. Schachtele J.M. Schaeken K.M. Schilling

R. Schmid U. Schroder P. Schüpbach A. Sheiham R.P. Shellis D.J. Smith L. Streebny J.W.

Stamm M. Steiner K.W.Stephen G.K. Stookey N. Strömberg L. Stösser J. Suhonen J. J. de Soet E. Söderling 
L. Tabak S. Takagi E. Tammisalo J. M. Tanzer W.-M. Tay T. Tervonen E.A. Thibodeau A. Thylstrup A.B. Tveit S. Twetman

W. Vann

M.H. van der Veen M. Vehkalahti R.M.H. Verbeeck E.H. Verdonschot

J. S. Wefel

S.H.YWei

A. Wenzel

K. Wennerholm

R.A. Whiley

D.J. White

G.M.Whitford

R.F.Wilson

J. Wolf

J.H.M. Wöltgens 